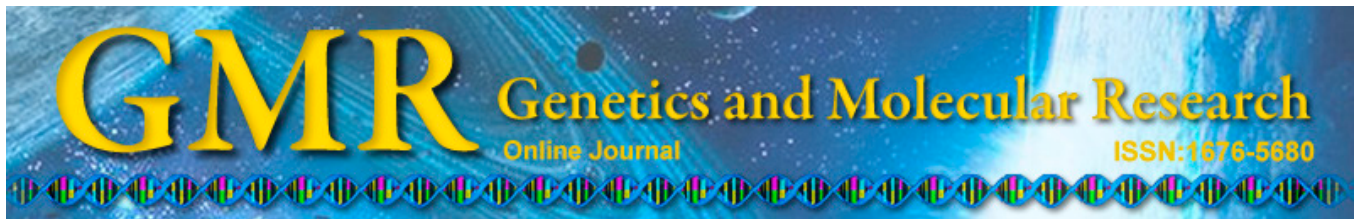

\title{
Identification of QTLs of resistance to white mold in common bean from multiple markers by using Bayesian analysis
}

\author{
L.A.C. Lara ${ }^{1}$, J.B. Santos ${ }^{2}$, M. Balestre ${ }^{3}$, I.A. Lima ${ }^{4}$, A.K.A. Pamplona ${ }^{3}$, \\ J.S. Veloso ${ }^{2}$ and P.H. Silva ${ }^{5}$ \\ ${ }^{1}$ Departamento de Genética, Universidade de São Paulo, Piracicaba, SP, Brasil \\ ${ }^{2}$ Departamento de Biologia, Universidade Federal de Lavras, Lavras, MG, Brasil \\ ${ }^{3}$ Departamento de Estatística, Universidade Federal de Lavras, Lavras, MG, \\ Brasil \\ ${ }^{4}$ Dupont Pioneer, Brasília, DF, Brasil \\ ${ }^{5}$ Embrapa Mandioca e Fruticultura, Cruz das Almas, BA, Brasil
}

Corresponding author: L.A.C. Lara

E-mail: leticia.lara.lac@gmail.com

Genet. Mol. Res. 14 (1): 1124-1135 (2015)

Received May 24, 2014

Accepted October 8, 2014

Published February 6, 2015

DOI http://dx.doi.org/10.4238/2015.February.6.16

\begin{abstract}
In this study, we identified simple sequence repeat, amplified fragment length polymorphism, and sequence-related amplified polymorphism markers linked to quantitative trait loci (QTLs) for resistance to white mold disease in common bean progenies derived from a cross between lines CNFC 9506 and RP-2, evaluated using the oxalic acid test and using Bayesian analysis. DNA was extracted from $186 \mathrm{~F}_{2}$ plants and their parental lines for molecular analysis. Fifteen experiments were carried out for phenotypic analysis, which included $186 \mathrm{~F}_{2: 4}$ progenies, the $\mathrm{F}_{1}$ generation, the $\mathrm{F}_{2}$ generation, and the lines CNFC 9506, RP-2, and G122 as common treatments. A completely randomized experimental design with 3 replications was used in controlled environments. The adjusted means for the $\mathrm{F}_{2: 4}$ generation were to identify QTLs by Bayesian shrinkage analysis. Significant differences were observed among the progenies for the reaction to white mold. The moving away method under the Bayes-
\end{abstract}


ian approach was effective for identifying QTLs when it was not possible to obtain a genetic map because of low marker density. Using the Wald test, 25 markers identified QTLs for resistance to white mold, as well as 16 simple sequence repeats, 7 amplified fragment length polymorphisms, and 2 sequence-related amplified polymorphisms. The markers BM184, BM211, and PV-gaat001 showed low distances from QTLs related white mold resistance. In addition, these markers showed, signal effects with increasing resistance to white mold and high heritability in the analysis with oxalic acid, and thus, are promising for marker-assisted selection.

Key words: Bayesian shrinkage analysis; Common bean; Plant breeding; Quantitative trait loci; Sclerotinia sclerotiorum

\section{INTRODUCTION}

The plant pathogen Sclerotinia sclerotiorum (Lib.) de Bary has gained attention in agricultural research because it is responsible for the occurrence of significant harvest losses in numerous countries (Silva et al., 2009). This pathogen is disseminated throughout diverse countries worldwide (Bolton et al., 2006; Schwartz and Singh, 2013) and its damages are manifested with greater severity in areas with mild temperatures, which is associated with high relative humidity.

Currently, there is no efficient method for controlling S. sclerotiorum. Therefore, there is a need for an integrated management plan that includes modifications in the growing method, application of fungicide, and the use of resistant cultivars. The use of resistant cultivars is recommended for disease management. However, this resistance is restricted to some white common bean cultivars of Mesoamerican origin and of Andean origin (Oliveira et al., 2005), cultivars which are not directly used by producers. There are also some lines and cultivars adapted to the conditions of the Center-West and Southeast of Brazil with partial resistance (Gonçalves and Santos, 2010; Schwartz and Singh, 2013).

Studies examining the molecular mechanisms of S. sclerotiorum pathogenicity have concentrated on enzymes that degrade the cell wall and produce oxalic acid. This acid penetrates the tissue around the lesion and acts directly on the host's organism. Oxalic acid production is therefore an important factor in the S. sclerotiorum infection process (Durman et al., 2005; Guimarães and Stotz, 2004; Kim et al., 2008).

It may be possible to evaluate the disease using an indirect method in which the cultivars/progenies are examined for their reaction to oxalic acid (Kolkman and Kelly, 2003; Antonio et al., 2008). The oxalic acid reaction test is very useful because it allows for quick discrimination of genotypes without the interference of the plant habitat on disease development (Gonçalves and Santos, 2010).

Various quantitative trait loci (QTLs) have been shown to provide the plant with resistance/tolerance to this pathogen (Mkwaila et al., 2011; Soule et al., 2011; Pérez-Vega et al., 2012; Miklas et al., 2013); however, it is difficult to build reliable genetic maps using molecular markers associated with these QTLs that are useful for marker-assisted selection.

Recently, some mapping techniques have allowed for concurrent identification of QTLs in the genome, improving the understanding of the genetic structure of the trait. The detection and localization of these QTLs are possible when molecular markers that are densely distributed throughout the genome are used. The interval mapping method has been widely 
used for this purpose. In this approach, the genotype of the QTL is not observable, but may be predicted based on bordering markers, i.e., markers defining an interval that may or may not contain a QTL (Lander and Botstein, 1989).

However, when there are few markers and low variability, QTLs located near a specific interval force the model to identify "ghost" QTLs because of the linkage of the actual QTL (present in adjacent intervals) with the interval being tested (Wu et al., 2007). In addition, if the genome is poorly saturated and innumerable QTLs are identified, the model must be rewritten to estimate the effects of the fixed positions on the estimates (Wang et al., 2005).

"Moving away" analysis by using likelihood inference was suggested by Doerge et al. (1997), which allows the search for QTLs with individual markers without the need for linkage groups. The main restriction of this method is that likelihood estimation is very restrictive for handling multiple effects. However, moving away can be evaluated under the Bayesian approach, allowing the search for multiple markers and simultaneous search for multiple QTLs. Thus, it is assumed that each QTL is a random variable derived from a normal distribution with a mean of 0 and individual variance. Thus, each possible QTL is penalized by the ratio of its variance to residual variance, where QTLs of small effect and low variability have their effects reduced to values near 0; in contrast, QTLs with large effects and high variability are less penalized by residual variance (Wang et al., 2005). Similar approaches have been developed by Xu (2003) and adapted by Wang et al. (2005), who added new parameters for genotype analysis using a QTL and its position in the genome.

Thus, the aim of this study was to identify simple sequence repeat (SSR), amplified fragment length polymorphism (AFLP), and sequence-related amplified polymorphism (SRAP) markers linked to QTLs for white mold resistance in common bean by using oxalic acid test, including controlled crosses and Bayesian shrinkage analysis for multiple markers.

\section{MATERIAL AND METHODS}

\section{Genotypes evaluated}

To obtain the $\mathrm{F}_{2}$ population, the lines CNFC 9506 and RP-2 were crossed. Based on their reaction to oxalic acid, these parental lines can be considered susceptible and partially resistant to white mold, respectively. According to the scoring scale proposed by Kolkman and Kelly (2000), the CNFC 9506 line received a score of 4.83 and the RP-2 line received a score of 1.97 (Gonçalves and Santos, 2010). The CNFC 9506 line was developed by Embrapa Arroz e Feijão and the RP-2 line by Universidade Federal de Lavras (UFLA). Both exhibit upright growth habit and carioca (beige with brown stripes) type grains.

\section{Phenotypic evaluation by oxalic acid}

A total of $186 \mathrm{~F}_{2: 4}$ progenies, the CNFC 9506 and RP-2 parents, and the G122 line were evaluated. The G122 line is a known source of resistance to white mold and has been shown to be moderately resistant, according to Gonçalves and Santos (2010). Each progeny and the 3 lines were evaluated in a plot composed of 10 plants. A completely randomized experimental design with 3 replications was used.

Initially, 60 seeds from each treatment were sown in a Styrofoam tray containing the substrate Plantmax, and the 30 most-uniform plants were selected for the experiment. Plants at 
the age of approximately 21 days were used, which is the age at which the second trifoliate leaf arises. Because of space limitations, 15 experiments were performed, including the evaluation of $13 \mathrm{~F}_{2: 4}$ progenies in 14 experiments, and, in the 15 th experiment, $4 \mathrm{~F}_{2: 4}$ progenies, $\mathrm{F}_{1}$, and $\mathrm{F}_{2}$ were evaluated. In addition to these genotypes, CNFC 9506, RP-2, and G122 were used as a common treatment in all experiments.

The experiments were conducted in an oxalic acid solution, as suggested by Kolkman and Kelly (2000), with some modifications. They were set up in temperature-controlled environments $\left(22^{\circ}-24^{\circ} \mathrm{C}\right)$ and the plants were acclimated to this environment before being cut. In the afternoon, the plants were cut at the base of the stem. The lower part of the stem was immersed in $20 \mathrm{mM}$ oxalic acid solution and kept in a plastic container for $21 \mathrm{~h}$ in a protected environment. The solution $\mathrm{pH}$ was 4.0 , adjusted using $\mathrm{NaOH}$. The plants under control treatment without oxalic acid were placed in a container with distilled water and a $\mathrm{pH}$ of 4.0.

After $21 \mathrm{~h}$ of exposition, the genotypes were evaluated for their reaction to oxalic acid using the descriptive scoring scale proposed by Kolkman and Kelly (2000).

\section{Genotypic evaluation}

DNA was extracted from the parents, CNFC 9506 and RP-2, and from the $186 \mathrm{~F}_{2}$ progenies according to the procedures described by Rodrigues and Santos (2006). Nucleic acids were rehydrated in TE buffer and quantified by $1 \%$ agarose gel electrophoresis using DNA markers of known concentrations. The quantified material was then diluted in pure water to a concentration of $10 \mathrm{ng} / \mu \mathrm{L}$ for use in polymerase chain reactions.

Prior to this, random primers for SSRs, AFLPs, and SRAPs were used and the polymorphic primers among the parents were selected. A total of 17 SSRs, 31 AFLPs, and 11 SRAPs were selected. These primers were used to genotype the $\mathrm{F}_{2}$ population of 186 plants.

The amplification products were subjected to denaturing polyacrylamide gel electrophoresis, stained in silver nitrate, and photographed using a digital camera.

The genotypes of the SSR markers were scored as $-1,0$, or 1 for the genotype of the smallest number of base pairs, heterozygote, and genotype of the greatest number of base pairs, respectively. The AFLP and SRAP markers were given scores 0 and 1, representing the absence and presence of the band, respectively.

\section{Bayesian shrinkage analysis}

The "moving away from marker" analysis was performed based on the conditional probabilities of the QTLs given a reference marker and the effects of these supposed QTLs were jointly included in the model. Therefore, we did not include or exclude markers during Markov chain Monte Carlo (MCMC) analysis. Thus, the linear model used was:

$$
y_{i}=b_{0}+\sum_{j}^{m} x_{i j} a_{j}+e_{i j}
$$

where $y_{i}$ is the corrected mean value of the $\mathrm{i}$-th progeny, $b_{0}$ is the overall mean value of the population under study, $m$ is the total number of markers, $x_{i j}$ is the representative variable that 
describes the genotype of the QTL, $a_{j}$ is the effect of the QTL associated with the $j$ marker, and $e_{\mathrm{ij}}$ is the residue assumed as $\mathrm{N}\left(0, \sigma_{e}^{2}\right)$.

In this model, $a_{j}$ is assumed to have a normal distribution with a mean value of 0 a variance of $\sigma_{a_{j}}^{2}$. The observable variables were the corrected mean values $\boldsymbol{y}=\left\{\mathrm{y}_{\mathrm{i}}\right\}$ for $i=1, \ldots$, $\mathrm{n}$ observations, and the genotypes of the markers $(m)$, while the non-observable variables were the effects of the QTLs $(a)$, their genotypes $\left(x_{i j}\right)$, its variances $\left(\sigma_{a_{j}}^{2}\right)$, and the residual variance $\left(\sigma_{e}^{2}\right)$. The priors are taken as follows:

$$
p\left(b_{0}\right) \propto 1, p\left(a_{j}\right) \propto N\left(0, \sigma_{a_{j}}^{2}\right), p\left(\sigma_{a_{j}}^{2}\right) \propto 1 / \sigma_{a_{j}}^{2} e p\left(\sigma_{e}^{2}\right) \propto 1 / \sigma_{e}^{2} \quad \text { (Equation 2) }
$$

Assuming independence among the parameter distributions and $b=\left\{b_{0}, a_{i}\right\}$ and $v=$ \{\} , the joint prior distribution of the non-observable variables $p(b, v)$ is the product of the priori distributions of individual parameters. The likelihood of the observable and non-observable variables is given as follows:

$$
p(\boldsymbol{y} \mid \boldsymbol{b}, \boldsymbol{v})=\prod_{i=1}^{n} p\left(y_{i} \mid \boldsymbol{b}, \sigma_{e}^{2}\right) \propto\left(\sigma_{e}^{2}\right)^{-n / 2} \exp \left\{-\frac{1}{2 \sigma_{e}^{2}} \sum_{i=1}^{n}\left(y_{i}-b_{0}-\sum_{j=1}^{m} x_{i j} a_{j}\right)^{2}\right\}
$$

Joint posteriori distribution is as follows:

$$
p(\boldsymbol{b}, \boldsymbol{v} \mid \boldsymbol{y}) \propto p(\boldsymbol{y} \mid \boldsymbol{b}, \boldsymbol{v}) p(\boldsymbol{b}, \boldsymbol{v})
$$

(Equation 4)

The genotypes are non-observable (missing information), but they may be inferred based on the information from the marker and from the positions $\left(\lambda_{i} \mathrm{~s}\right)$ of the QTLs in relation to the marker. In this study, we assumed that each marker was linked to a QTL, such that $\lambda_{j}$ was uniformly distributed between 2 intervals corresponding to a recombination frequency ranging from 0 (mark is the QTL) to 0.5 (independent segregation between the marker and QTL) (Balestre, 2012).

$$
p(\boldsymbol{b}, \boldsymbol{v}, \lambda)=p\left(b_{0}\right) p\left(\sigma_{e}^{2}\right) \prod_{j=1}^{m} p\left(\lambda_{j}\right) p\left(a_{j}\right) p\left(\sigma_{a_{j}}^{2}\right)
$$

Assuming independence among the effects and variance and the genotypes of the QTLs, as well as the independence of the observations in relation to the markers and their genetic distances, we determined the likelihood by using the following:

$$
p(\boldsymbol{b}, \boldsymbol{v}, \boldsymbol{x}, \lambda \mid \boldsymbol{y}, \boldsymbol{m}) \propto p(\boldsymbol{y} \mid \boldsymbol{b}, \boldsymbol{v}, \boldsymbol{x}) p(\boldsymbol{x} \mid \lambda, \boldsymbol{m}) p(\boldsymbol{b}, \boldsymbol{v}, \lambda)
$$

(Equation 6)

In $\mathrm{F}_{2: 4}$ populations, the probability of heterozygous plants within each family was given by 0.125 .

Each QTL genotype was sampled from a multinomial distribution, with probability determined using: 


$$
p\left(x_{i j} \mid \lambda_{j}, m_{j}, \boldsymbol{y}\right)=\frac{p\left(x_{i j} \mid \lambda_{j}, m_{j}\right) p\left(y_{i j} \mid \boldsymbol{b}, \sigma_{e}^{2}, x_{i j}\right)}{\sum_{z=\{-1,0,1\}} p\left(x_{i j}=z \mid \lambda_{j}, m_{j}\right) p\left(y_{i j} \mid \boldsymbol{b}, \sigma_{e}^{2}, x_{i j}=z\right)} \quad \text { (Equation 7) }
$$

The $\lambda$ parameter does not have a known function for the density of a posteriori conditional probability, and the use of the Gibbs sampler is not possible because it uses an iterative process with a known distribution, taking samples from a Markov chain. Another MCMC method may be used for sampling, known as the Metropolis-Hastings method (Metropolis et al., 1953; Hastings, 1970). This method does not require the parameter to have a known probability function. In the method presented, a uniform distribution may be used as an auxiliary function, where a new position is sampled $\left(\lambda^{*}\right)$, using the Haldane function, over an interval delimited by $\max \left(0, r_{j}-d\right)$ and $\min \left(0.5, r_{j}+d\right)$, where $d$ is a constant that defines the pathway within interval $j$, normally a value of 1 or $2 \mathrm{cM}$. This function is denoted by $\left(\lambda^{*}, \lambda\right)$, and the new position will be accepted in the k-th interaction with $\min (1, \alpha)$ of probability, where:

$$
\alpha=\frac{p\left(\lambda_{j}^{*} \mid \boldsymbol{y}, b_{j}, \sigma_{e}^{2}\right) u\left(\lambda_{j}^{*}, \lambda_{j}\right)}{p\left(\lambda_{j}^{0} \mid \boldsymbol{y}, b_{j}, \sigma_{e}^{2}\right) u\left(\lambda_{j}, \lambda_{j}^{*}\right)}
$$

Thus, if $\alpha$ is accepted, a new position is established and a new genotype is suggested for the $x$ matrix of genotypes of the QTLs, closing an MCMC cycle (Satagopan et al., 1996; Wang et al., 2005; Banerjee et al., 2008). The other a posteriori conditional distributions for the $b$ and $v$ parameters are similar to those described by $\mathrm{Xu}$ (2003).

\section{Post-MCMC analysis}

The aim of Bayesian analysis through MCMC is to obtain an empirical a posteriori distribution from which all information with respect to the QTL may be obtained. Thus, the significance test is not very important in Bayesian inference, as in likelihood analysis. In simple Bayesian analysis, the QTL position is inferred based on the number of times that the effect of the QTL passes through a small region (bin) in a determined position of the genome. This curve describes the intensity profile of the QTL. Based on the approach of Wang et al. (2005), it is assumed that each interval is associated with a QTL, such that in all the intervals, the supposed QTL will pass through all regions of the genome, and, in each interval, the same number of hits of the QTL will be observed regardless of its effect. Nevertheless, a true QTL is expected to occur in a given interval and its position will show a peak, whereas if the effect is null, the distribution within the interval is uniform (Yang and $\mathrm{Xu}, 2007$ ). The profile intensity of the QTL was described by Yang and Xu (2007) as a function of the position. However, it may not be sufficiently informative for inference regarding QTL in Bayesian shrinkage analysis. Based on this, Yang and $\mathrm{Xu}$ (2007) described the effects of QTLs according to their quadratic forms, weighted by the position intensity: $g(\lambda)=W(\lambda) f(\lambda)$, with $W(\lambda)=a V_{a}^{-1} a$, where $V_{a}^{-1}$ is the

inverse of the variances of the effects of the QTLs, given by $\left(\sum_{i=1}^{n} x_{j}^{2}+\sigma_{e}^{2} / \sigma_{a_{1}}^{2}\right)^{-1} \sigma_{e}^{2}$, which corresponds to the information matrix of the effect. This test, known as the Wald test, follows a chi-square distribution with 2 degrees of freedom (Yang and $\mathrm{Xu}, 2007$ ). 


\section{RESULTS}

\section{Identification of QTLs}

The detection of QTLs associated with resistance to white mold using evaluation of oxalic acid is shown in Figure 1 and Table 1. The scale of values of the Wald test is on the ordinate and the representation of the markers is on the abscissa, where 1-17 indicate SSR markers, 18-48 are AFLP markers, and 49-59 represent SRAP markers.

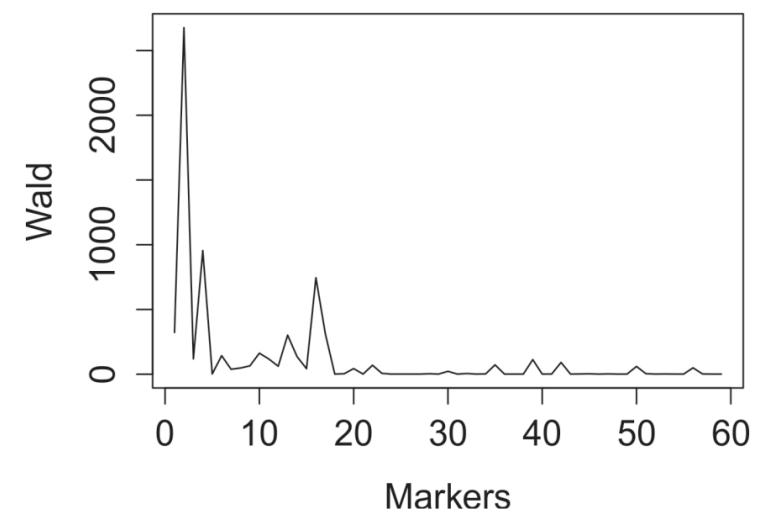

Figure 1. Analysis of the SSR, AFLP, and SRAP markers to identify the QTLs of resistance to white mold by using the Bayesian shrinkage procedure, with 10,000 iterations.

Table 1. Summary of the distance between the marker and QTL, position of the markers in the figures, effect of the QTL associated with the marker, and the respective magnitudes and heritability of the QTL.

\begin{tabular}{|c|c|c|c|c|c|c|}
\hline Marker & Position & $\mathrm{FR}^{\mathrm{a}}(\%)$ & Distance $^{\mathrm{b}}$ & Effect & Wald $^{\mathrm{c}}$ & $\mathrm{h}^{2 \mathrm{~d}}(\%)$ \\
\hline BM184 & 1 & 3.34 & 3.34 & 0.108 & 322.43 & 54.85 \\
\hline BM187 & 2 & 35.24 & 43.84 & -0.316 & 2676.59 & 85.87 \\
\hline BM211 & 3 & 1.61 & 1.61 & 0.058 & 118.5 & 75.99 \\
\hline BMd42a & 4 & 26.84 & 29.98 & 0.187 & 954.82 & 66.65 \\
\hline BM165 & 6 & 2.07 & 2.07 & 0.029 & 142.89 & 2.55 \\
\hline BM212 & 7 & 2.36 & 2.36 & 0.013 & 38.43 & 0.02 \\
\hline PV188 & 8 & 1.74 & 1.74 & 0.025 & 47.97 & 37.48 \\
\hline PV74 & 9 & 1.91 & 1.91 & -0.012 & 64.8 & 54.27 \\
\hline PVESTBR_185 & 10 & 7.48 & 7.54 & -0.029 & 162.55 & 72.25 \\
\hline PVESTBR_204 & 11 & 2.05 & 2.05 & 0.051 & 117.69 & 66.95 \\
\hline PVESTBR_42 & 12 & 2.43 & 2.43 & 0.026 & 61.65 & 47.83 \\
\hline PV-gaat001- & 13 & 2.13 & 2.13 & 0.085 & 301.62 & 82.86 \\
\hline ATA244 & 14 & 1.87 & 1.87 & 0.033 & 135.58 & 1.01 \\
\hline ME1 & 15 & 2.29 & 2.29 & 0.022 & 42.67 & 0.55 \\
\hline BMc94 & 16 & 38.12 & 50.1 & -0.074 & 745.2 & 83.8 \\
\hline $\mathrm{BMc} 83$ & 17 & 2.61 & 2.61 & -0.103 & 310.87 & 79.08 \\
\hline $\mathrm{EAGG} / \mathrm{MCAG}_{252}$ & 20 & 9.77 & 9.9 & -0.017 & 43.35 & 0.45 \\
\hline EAAG/MCAG ${ }_{447}^{252}$ & 22 & 12.92 & 13.22 & -0.022 & 69.14 & 2.03 \\
\hline $\mathrm{EAAG} / \mathrm{MCAG}_{398}$ & 23 & 2.01 & 2.01 & -0.002 & 6.58 & 4.02 \\
\hline $\mathrm{EAGA} / \mathrm{MCAG}_{186}^{398}$ & 30 & 7.82 & 7.88 & -0.011 & 22.39 & 8.27 \\
\hline $\mathrm{EACC} \mathrm{MCAT}_{282}{ }_{286}$ & 35 & 2.13 & 2.13 & -0.022 & 72.36 & 2.62 \\
\hline $\mathrm{EACA} / \mathrm{MCAT}_{700}^{282}$ & 39 & 2.04 & 2.04 & 0.028 & 112.73 & 3.27 \\
\hline $\mathrm{EACA} / \mathrm{MCAT}_{447}$ & 42 & 11.87 & 12.1 & 0.024 & 90.94 & 1.34 \\
\hline $\mathrm{Me} 9 \mathrm{~F} / \mathrm{Em} 7 \mathrm{R}_{700}$ & 50 & 4.32 & 4.33 & -0.02 & 60.09 & 0.82 \\
\hline $\mathrm{Me} 9 \mathrm{~F} / \mathrm{Em} 7 \mathrm{R}_{173}$ & 56 & 1.42 & 1.42 & -0.018 & 49.23 & 2.4 \\
\hline
\end{tabular}

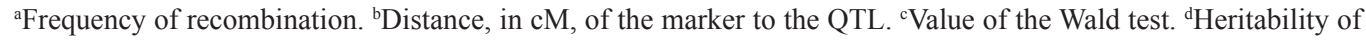
the QTL; $(-)=$ reduced resistance. 
Twenty-five markers identified QTLs with resistance to white mold, including 16 SSRs (BM184, BM187, BM211, BMd42A, BM165, BM212, PV188, PV74, PVESTBR_185, PVESTBR_204, PVESTBR_42, PV-gaat001, ATA244, ME1, BMc94, and BMc83), 7 AFLPs $\left(\mathrm{EAGG} / \mathrm{MCAG}_{252}, \mathrm{EAAG} / \mathrm{MCAG}_{447}, \mathrm{EAAG} / \mathrm{MCAG}_{398}, \mathrm{EAGA} / \mathrm{MCAG}_{186}, \mathrm{EACC} / \mathrm{MCAT}_{282}\right.$, $\mathrm{EACA}_{\mathrm{MCAT}}{ }_{700}$, and $\left.\mathrm{EACA} / \mathrm{MCAT}_{447}\right)$, and $2 \mathrm{SRAPs}\left(\mathrm{Me} 9 \mathrm{~F} / \mathrm{Em} 7 \mathrm{R}_{700}\right.$ and $\mathrm{Me} 9 \mathrm{~F} / \mathrm{Em} 7 \mathrm{R}_{173}$ ). Of these, only BM187, BMd42a, and BMc94 were associated with highly significant QTLs according to the Wald test.

The effect of the QTLs on the expression of resistance to white mold is shown in Figure 2. On the ordinate is the effect, ranging from -0.3 (contributed to reducing resistance) to 0.2 (contributed to increasing resistance). Representation of the markers is on the abscissa.

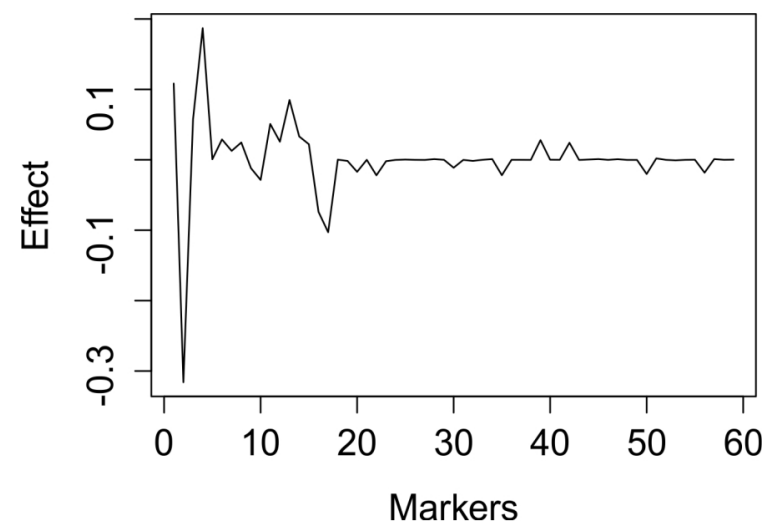

Figure 2. Effect of the QTL associated with the marker, with 10,000 iterations.

Among the 25 markers, 13 were linked to the QTLs, with effects of increasing resistance to white mold. These markers included BM184, BM211, BMd42a, BM165, BM212, PV188, PVESTBR_204, PVESTBR_42, PV-gaat001, ATA244, ME1, MCAT/EACA ${ }_{700}$, and $\mathrm{MCAT} / \mathrm{EACA}_{447}$. Of these, only 1 was related to a highly significant QTL (BMd42a).

The frequency of recombination of the QTL with the marker is shown in Figure 3 and Table 1. The frequency of recombination is on the ordinate and the representation of the markers is on the abscissa.

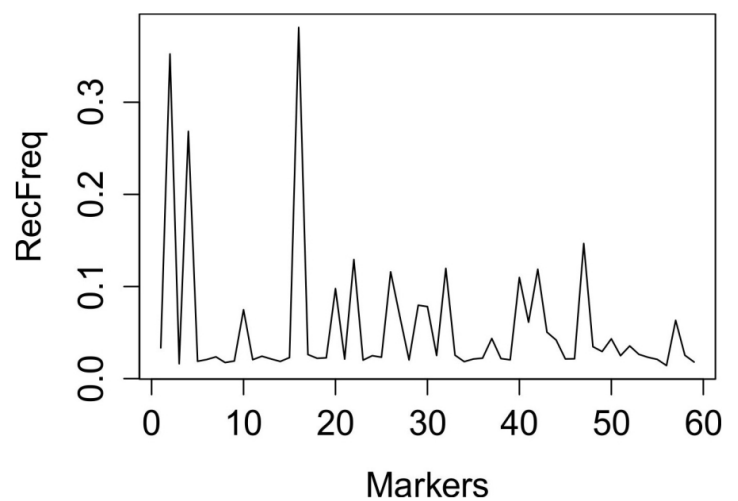

Figure 3. Frequency of recombination of the markers, with 10,000 iterations. 
The markers BM187, BMd42a, and BMc94 segregated nearly independently from the associated QTLs because they showed a high frequency of recombination (from 26.84 to $38.12 \%$ ) (Table 1, Figure 3).

The summary of the distance data, in cM, between the marker and the QTL, the position of the marker in the figures, and the effect and heritability of the QTL associated with resistance to white mold through evaluation in oxalic acid are shown in Table 1.

Among the microsatellite markers evaluated, only PVM02TC116 did not identify a QTL for resistance to white mold.

\section{DISCUSSION}

The BM184 marker was initially mapped in LG 11 (Blair et al., 2003, 2008). However, Maxwell et al. (2007) identified this marker in LG 9, evaluating the RIL G122/CO72548 population. This marker is also related to the QTLs for days to flowering, pods per plant, and 100 seed weight (Blair et al., 2006). In this study, the BM184 marker identified a QTL affecting increasing resistance to white mold at a distance of $3.34 \mathrm{cM}$ from the QTL. This QTL showed moderate heritability of $54.85 \%$, which measures the reliability of marker-assisted selection (MAS) as it determines the percentage of genetic variation that will be inherited.

The BM187 marker was mapped in LG 6, in which only 1 QTL for resistance to white mold was previously identified, WM6.1 ${ }^{\mathrm{B} 60, \mathrm{R} 31}$, first identified in the RIL Benton/NY6020-4 population (Miklas et al., 2003) and then in the RIL Raven/I9365-31 population (Soule et al., 2011). This same marker, according to Blair et al. (2006), flanks QTLs for days to flowering, plant height, plant width, and number of seeds per plant and for the $\mathrm{V}$ gene for flower color. In this study, the BM187 marker identified the most significant QTL for the effect of reducing resistance to white mold. The QTL showed high heritability $(85.87 \%)$; however, it was located far from the marker $(43.84 \mathrm{cM})$.

The BM211 marker separated 2 QTLs in LG 8 (Park et al., 2001; Maxwell et al., 2007). This linkage group contains 4 QTLs already identified for resistance to white mold. Grisi et al. (2007) also identified the BM165 marker in LG 8, associated with 1 of the 4 QTLs for resistance to white mold. In this study, the BM211 and BM165 markers were found near the QTLs, at $1.61 \mathrm{cM}$ and $2.07 \mathrm{cM}$, respectively. Both QTLs increase resistance to white mold. The heritability of the QTL linked to the BM211 marker is high, at $75.99 \%$, and heritability of the QTL linked to the BM165 marker is low, at 2.55\%. Therefore, only the BM211 marker is promising for MAS because it identifies a highly heritable QTL.

The PV-gaat001 marker is linked to a QTL as it increases resistance to white mold. This marker was initially mapped by Yu et al. (2000) in 2 RIL populations, DOR364/G19833 and BAT93/JaloEEP558, in LG 4, and has frequently been reported in the literature (Blair et al., 2003, 2008; Mkwaila et al., 2011; Soule et al., 2011; Miklas et al., 2013). In its linkage group, there are 2 QTLs-WM4.1 ${ }^{\mathrm{PX}}$ and WM4.2 ${ }^{\mathrm{R} 31}$, identified by Park et al. (2001) and Soule et al. (2011), respectively. The PV-gaat001 marker was effective for identifying a QTL by using the oxalic acid method, at $2.13 \mathrm{cM}$ from the QTL. This QTL showed high heritability $(82.86 \%)$, and is promising for use in MAS.

The ME1 marker was initially marked in LG 1 by Blair et al. (2008) and then reported in LG 9, and is present in the linkage map published by Galeano et al. (2009) and Blair et al. (2010). In this study, ME1 was found near a QTL $(2.29 \mathrm{cM})$ and showed an effect of increasing resistance to white mold, but with low heritability $(0.55 \%)$. 
The BMd42a, BM212, and ATA244 markers evaluated in this study have appeared in the literature for LG 10 (Freyre et al., 1998; Grisi et al., 2007; Miklas et al., 2013). All identified QTLs increased the resistance to white mold. Of these, only the BMd42a marker was quite far from the QTL, at $29.98 \mathrm{cM}$, and showed $66.65 \%$ heritability. The BM212 and ATA244 markers, despite being near the QTLs identified (at 2.36 and $1.87 \mathrm{cM}$, respectively), are not promising for MAS because they showed low heritability $(0.02$ and $1.01 \%$, respectively). According to Blair et al. (2006), the BM212 marker is also near a QTL for 100 seed weight.

The markers PV188, PV74, PVESTBR_185, PVESTBR_204, PVESTBR_42, BMc94, and BMc83 were significant for identifying QTLs related to resistance to white mold. Of these, BMc94, despite being a highly significant QTL with high heritability (83.8\%), segregated independently from this QTL (50.10 cM distance), and is thus not promising for MAS. The markers PVESTBR_185 and BMc83 identified QTLs that reduced resistance to white mold and were relative close to the QTL (7.54 and $2.61 \mathrm{cM}$, respectively); these QTLs showed high heritability (72.25 and 79.08\%, respectively). These markers, together with the AFLPs $\left(\mathrm{EAGG} / \mathrm{MCAG}_{252}, \mathrm{EAAG} / \mathrm{MCAG}_{447}, \mathrm{EAAG} / \mathrm{MCAG}_{398}, \mathrm{EAGA} / \mathrm{MCAG}_{186}, \mathrm{EACC} / \mathrm{MCAT}_{282}\right.$, $\left.\mathrm{EACA} \mathrm{MCAT}_{700}, \mathrm{EACA} \mathrm{MCAT}_{447}\right)$ and the SRAPs $\left(\mathrm{Me} 9 \mathrm{~F} / \mathrm{Em} 7 \mathrm{R}_{700}\right.$ and $\mathrm{Me} 9 \mathrm{~F} / \mathrm{Em} 7 \mathrm{R}_{173}$ ), have not been reported previously. Notably, all AFLP and SRAP markers identified QTLs of low heritability, and thus were not promising for MAS in evaluations using the oxalic acid absorption method.

In this study, the moving away method under the Bayesian approach was found to be efficient for identifying QTLs when it is not possible to obtain a genetic map because of the low density of markers. The disadvantage of this approach is the lack of exact identification of the QTL position in the genome, as the search is pivotal and not based on intervals as for classical interval markers.

The markers BM184, BM211, and PV-gaat001 are near QTLs with the effect of increasing resistance to white mold and of high heritability in analysis with oxalic acid, and thus are promising for use in MAS.

\section{ACKNOWLEDGMENTS}

Research supported by Conselho Nacional de Desenvolvimento Científico e Tecnológico $(\mathrm{CNPq})$, Fundação de Amparo à Pesquisa do Estado de Minas Gerais (FAPEMIG), and Coordenação de Aperfeiçoamento de Pessoal de Nível Superior (CAPES).

\section{REFERENCES}

Antonio RP, Santos JB, Souza TP and Carneiro FF (2008). Genetic control of the resistance of common beans to white mold using the reaction to oxalic acid. Genet. Mol. Res. 7: 733-740.

Balestre M (2012). Mapeamento Bayesiano de Múltiplos Caracteres: Efeitos Pleiotrópicos no Estudo da Herança dos Componentes da Produção em Milho. Doctoral thesis, UFLA, Lavras.

Banerjee S, Yandell BS and Yi NJ (2008). Bayesian quantitative trait loci mapping for multiple traits. Genetics. 179: 2275-2289.

Blair MW, Pedraza F, Buendia HF, Gaitan-Solis E, et al. (2003). Development of a genome-wide anchored microsatellite map for common bean (Phaseolus vulgaris L.). Theor. Appl. Genet. 107: 1362-1374.

Blair MW, Iriarte G and Beebe S (2006). QTL analysis of yield traits in an advanced backcross population derived from a cultivated Andean x wild common bean (Phaseolus vulgaris L.) cross. Theor. Appl. Genet. 112: 1149-1163. 
Blair MW, Buendia HF, Giraldo MC, Métais I, et al. (2008). Characterization of AT-rich microsatellites in common bean (Phaseolus vulgaris L.). Theor. Appl. Genet. 118: 91-103.

Blair MW, Medina JI, Astudillo C, Rengifo J, et al. (2010). QTL for seed iron and zinc concentration and content in a Mesoamerican common bean (Phaseolus vulgaris L.) population. Theor. Appl. Genet. 121: 1059-1070.

Bolton MD, Thomma BP and Nelson BD (2006). Sclerotinia sclerotiorum (Lib.) de Bary: biology and molecular traits of a cosmopolitan pathogen. Mol. Plant Pathol. 7: 1-16.

Doerge RW, Zeng Z-B and Weir BS (1997). Statistical issues in the search for genes affecting quantitative traits in experimental populations. Stat. Sci. 12: 195-219.

Durman SB, Menendez AB and Godeas AM (2005). Variation in oxalic acid production and mycelia compatibility within field populations of Sclerotinia sclerotiorum. Soil Biol. Biochem. 37: 2180-2184.

Freyre R, Skroch PW, Geffroy V and Adam-Blondon AF (1998). Towards an integrated linkage map of common bean. 4. Development of a core linkage map and alignment of RFLP maps. Theor. Appl. Genet. 97: 847-856.

Galeano CH, Fernández AC, Gómez M and Blair MW (2009). Single strand conformation polymorphism based SNP and Indel markers for genetic mapping and synteny analysis of common bean (Phaseolus vulgaris L.). BMC Genomics 10: 629 .

Gonçalves PRC and Santos JB (2010). Physiological resistance of common bean cultivars and lines to white mold based on oxalic acid reaction. Ann. Rep. Bean Improv. Coop. 53: 236-237.

Grisi MCM, Blair MW, Gepts P and Brondani C (2007). Genetic mapping of a new set of microsatellite markers in a reference common bean (Phaseolus vulgaris L.) population BAT93/JALO EEP558. Genet. Mol. Res. 3: 691-706.

Guimarães RL and Stotz HU (2004). Oxalate production by Sclerotinia sclerotiorum deregulates guard cells during infection. Plant Physiol. 136: 3703-3711.

Hastings WK (1970) Monte carlo sampling methods using markov chains and their applications. Biometrika. 57: 97-109.

Kim KS, Min JY and Dickman MB (2008). Oxalic acid is an elicitor of plant programmed cell death during Sclerotinia sclerotiorum disease development. Mol. Plant Microbe. Interact. 21: 605-612.

Kolkman JM and Kelly JD (2000). An indirect test using oxalate to determine physiological resistance to white mold in common bean. Crop Sci. 40: 281-285.

Kolkman JM and Kelly JD (2003). QTL conferring resistance and avoidance to white mold in common bean. Crop Sci. 43: 539-548.

Lander ES and Botstein D (1989). Mapping mendelian factors underlying quantitative traits using RFLP linkage maps. Genetics. 121: 185-199.

Maxwell JJ, Brick MA, Byrne PF, Schwartz HF, et al. (2007). Quantitative trait loci linked to white mold resistance in common bean. Crop Sci. 47: 2285-2294.

Metropolis N, Rosenbluth AW, Rosenbluth MN, Teller AH, et al. (1953) Equation of state calculations by fast computing machines. J. Chem. Phys. 21: 1087-1092.

Miklas PN, Delorme R and Riley R (2003). Identification of QTL conditioning resistance to white mold in snap bean. $J$. Am. Soc. Hortic. Sci. 128: 564-570.

Miklas PN, Porter LD, Kelly JD and Myers JR (2013). Characterization of white mold disease avoidance in common bean. Eur. J. Plant Pathol. 135: 525-543.

Mkwaila W, Terpstra KA, Ender M and Kelly JD (2011). Identification of QTL for agronomic traits and resistance to white mold in wild and landrace germplasm of common bean. Plant Breed. 130: 665-672.

Oliveira RP, Cristofani M and Machado MA (2005). Integrated genetic map of citrus base on RAPD markers. Fruits 60: 187-193.

Park SO, Coyne DP, Steadman JR and Skroch PW (2001). Mapping of QTL for resistance to white mold disease in common bean. Crop Sci. 41: 1253-1262.

Pérez-Vega E, Pascual A, Campa A, Giraldez R, et al. (2012). Mapping quantitative trait loci conferring partial physiological resistance to White mold in the common bean RIL population Xana x Cornell 49242. Mol. Breed. 29: 31-41.

Rodrigues TB and Santos JB (2006). Effect of natural selection on common bean microsatellite (SSR) alleles. Genet. Mol. Biol. 29: 345-352.

Satagopan JM, Yandell BS, Newton MA and Osborn TC (1996) A Bayesian approach to detect quantitative trait loci using Markov chain Monte Carlo. Genetics. 144: 805-816.

Schwartz HF and Singh SP (2013). Breeding common bean for resistance to white mold: A review. Crop Sci. 53: 18321844.

Silva AP, Bolton MD and Nelson BD (2009). Transformation of Sclerotinia sclerotiorum with the green fluorescent protein gene and fluorescence of hyphae in four inoculated hosts. ISRN Mol. Biol. 58: 487-496.

Soule M, Porter L, Medina J and Santana GP (2011). Comparative QTL map for white mold resistance in common bean, and characterization of partial resistance in dry bean lines VA19 and 19365-31. Crop Sci. 51: 123-139.

Genetics and Molecular Research 14 (1): 1124-1135 (2015)

CFUNPEC-RP www.funpecrp.com.br 
Wang H, Zhang YM, Li X and Masinde GL (2005). Bayesian shrinkage estimation of quantitative trait loci parameters. Genetics 170: 465-480.

Wu R, Ma CX and Casella G (2007). Statistical Genetics of Quantitative Traits: Linkage, Maps and QTL. Springer-Verlag, New York.

Xu S (2003). Estimating polygenic effects using markers of the entire genome. Genetics 163: 789-801.

Yang R and Xu S (2007) Bayesian shrinkage analysis of quantitative trait loci for dynamic traits. Genetics. 176: 11691185.

Yu K, Park SJ, Poysa V and Gepts P (2000). Integration of simple sequence repeat (SSR) markers into a molecular linkage map of common bean (Phaseolus vulgaris L.). J. Hered. 91: 429-434. 